\title{
Synaptic Protein Degradation Underlies Destabilization of Retrieved Fear Memory
}

Sue-Hyun Lee, Jun-Hyeok Choi, Nuribalhae Lee, Hye-Ryeon Lee, Jae-Ick Kim, Nam-Kyung Yu, Sun-Lim Choi, Seung-Hee Lee, Hyoung Kim, Bong-Kiun Kaang*

National Creative Research Initiative Center for Memory, Department of Biological Sciences, College of Natural Sciences, Seoul National University, San 56-1 Silim-dong, Gwanak-gu, Seoul 151-747, Korea.

*To whom correspondence should be addressed. E-mail: kaang@snu.ac.kr

Reactivated memory undergoes a rebuilding process which depends on de novo protein synthesis. This suggests that retrieval is dynamic and serves to incorporate new information into preexisting memories. However, little is known about whether or not protein degradation is involved in the reorganization of retrieved memory. We found that postsynaptic proteins were degraded in the hippocampus by poly-ubiquitination after retrieval of contextual fear memory. Moreover, the infusion of proteasome inhibitor into the CA1 region immediately after retrieval prevented the anisomycin-induced memory impairment, as well as the extinction of fear memory. This suggests that ubiquitin- and proteasome-dependent protein degradation underlies destabilization processes after fear memory retrieval. It also provides strong evidence for the existence of reorganization processes whereby preexisting memory is disrupted by protein degradation, and updated memory is reconsolidated by protein synthesis.

Memory retrieval is a process of recalling a previously stored memory. Recently, memory retrieval has attracted much attention because it has been found that inhibition of protein synthesis before or immediately after memory retrieval impairs the previously consolidated memory (1-4). Retrieval of a consolidated memory thus returns the memory storage site to a labile state, after which new protein synthesis is required for stabilizing or reconsolidating the memory (1-9). This suggests that the retrieval of the consolidated memory is a dynamic and active process in which remodeling or reorganization of the already-formed memories occurs to incorporate new information $(2,3,6)$.

Although it has attracted less attention than the gene transcription and protein synthesis model for long-lasting synaptic changes and memory stabilization, protein degradation is also critical for long-term memory (10-16). A major cellular mechanism controlling protein turnover is the ubiquitin/proteasome system, in which poly-ubiquitinated proteins are degraded by the multi-subunit proteasome complex $(11,17)$. A subunit of the $26 S$ proteasome, S5a which selectively binds to poly-ubiquitinated proteins, plays a critical role in protein degradation $(18,19)$.

If retrieval stimuli trigger new protein synthesis for the remodeling of consolidated memory, protein degradation via the ubiquitin/proteasome system might be necessary because remodeling of synapses, which encode the memory, would be mediated by removal of existing proteins and by incorporation of new proteins (11). However, little is known about the protein degradation mechanism during the reorganization process after memory retrieval in vivo. We therefore investigated the involvement of the ubiquitin/proteasome system and the roles of protein degradation during the destabilization/restabilization process after fear memory retrieval.

We first performed a total protein poly-ubiquitination assay after fear memory retrieval. Poly-ubiquitinated proteins were purified by immobilized fusion proteins of S5a (11) from the crude synaptosomal membranes of the hippocampi, which were isolated after contextual fear memory retrieval (Fig. 1A). The poly-ubiquitinated protein level in the $15 \mathrm{~min}$ group and the 60 min group whose hippocampi were isolated $15 \mathrm{~min}$ and $60 \mathrm{~min}$ after the beginning time-point of retrieval, respectively, increased remarkably, whereas the polyubiquitination level was little changed in the absence of retrieval (No R group) compared to 'Naïve' mice which received only handling (Fig. 1B). Reconsolidation is not affected by protein synthesis inhibitor when the retrieval duration is very short (up to $1 \mathrm{~min}$ re-exposure) (2). Thus, we examined the effect of retrieval duration on protein polyubiquitination. Anisomycin treatment following 1 min reexposure did not affect freezing level after $24 \mathrm{~h}$ (fig. S1, A and B). The poly-ubiquitinated protein level of the $1 \mathrm{~min}$ reexposure group was comparable to the level of No R group and was significantly lower than that of the $5 \mathrm{~min}$ re-exposure group (fig. S1, C and D). This indicates that protein polyubiquitination is increased specifically in response to retrieval signal that induces protein synthesis-dependent reconsolidation. 
We next asked which proteins are poly-ubiquitinated and degraded after fear memory retrieval in the hippocampus. It has been shown that neuronal activity induces the turnover and remodeling of several PSD proteins, which is critical for $\operatorname{LTP}(11,20)$. To examine whether proteins in PSD may be possible targets for degradation during retrieval, we performed immunoblot analyses with antibodies against three different PSD proteins: Shank, GKAP and PSD-95, by using GST-S5a pulldown assay shown in Fig. 1. Poly-ubiquitinated Shank and GKAP, but not PSD-95, increased remarkably $1 \mathrm{~h}$ after retrieval (Fig. 2, A and B). In the mouse hippocampus, multiple Shank bands which indicate the products of alternative splicing with diverse sizes ranging from $120 \mathrm{kDa}$ to $240 \mathrm{kDa}$ were observed as previously described in the rat cortex (21) (fig. S2). Interestingly, the protein level of 130 $\mathrm{kDa}$ and $160 \mathrm{kDa}$ endogenous Shank was decreased 1 2 h after retrieval in the synaptic region (Fig. 2, C to E). This decrease may reflect the ubiquitin/proteasome-dependent degradation process, as the time course of the increase of Shank poly-ubiquitination almost coincided with the time course of the reduction of the endogenous Shank level (Fig. 2, A and B). To elucidate this point more clearly, we infused either the ubiquitin/proteasome inhibitor clasto-Lactacystin$\beta$-lactone ( $\beta$ lac) or vehicle into area CA1. We found that $\beta$ lac infusion blocked the decrease of Shank level at $2 \mathrm{~h}$ after retrieval and kept the Shank level up to the basal level in control animals without retrieval (fig. S3). Thus, the dynamic change in the expression level of specific PSD proteins such as Shank and GKAP (Fig. 2, C to E) implies the possibility that synaptic destabilizing and restabilizing states are triggered after the memory retrieval process, as previously suggested $(1-3,6)$. Furthermore, the protein synthesis during the reconsolidation may be a compensatory mechanism for protein degradation induced by the retrieval.

The increase in poly-ubiquitinated proteins at the synapses during fear memory retrieval raises a question: what is the physiological function of protein degradation after memory retrieval? We bilaterally administered ßlac or the protein synthesis blocker anisomycin into the CA1 region immediately after the $1^{\text {st }}$ retrieval (Fig. 3, A and B; retrieval 1). The freezing levels were not significantly different among groups on retrieval 1 before the drug infusion (fig. S4). Then, we re-tested the fear level $24 \mathrm{~h}$ after the infusion (Fig. 3C; retrieval 2). We did not observe any difference between the vehicle control and the $\beta$ lac group whereas we could observe fear memory impairment at retrieval 2 of the anisomycin group as reported previously $(1,2)$ (Fig. 3, C and D). However, co-infusion of $\beta$ lac with anisomycin prevented the memory impairment caused by the single infusion of anisomycin at retrieval 2 (Fig. 3, C and D). The increase in protein degradation may contribute to both the destabilization of preexisting fear memory and the restabilization of reorganized fear memory (11-15). Our data support the idea that protein degradation after memory retrieval is important for the destabilization of preexisting fear memory, rather than for the restabilization process. If the major function of protein degradation after retrieval was removal of inhibitory proteins for the memory restabilization, $\beta$ lac infusion should have impaired the fear memory at retrieval $2(16,22,23)$. However, the infusion of $\beta$ lac alone did not affect the fear level. If the ubiquitin-proteasome pathway was involved in forgetting the preexisting fear memory, blocking ubiquitinproteasome pathway would have suppressed the anisomycininduced impairment of fear memory retrieval by inhibiting the destruction process of the preexisting fear memory (8). Consistent with this idea, we found that the co-infusion of Blac with anisomycin was able to prevent memory impairment caused by anisomycin following retrieval (Fig. 3, $\mathrm{C}$ and $\mathrm{D}$ ). This indicates that $\beta$ lac may inhibit the destruction process of the previously formed fear memory and maintain the freezing level even when the reconsolidation is inhibited without new protein synthesis.

To further support this idea, we examined the effect of Blac infusion during fear memory acquisition (Fig. 4A). Because there is no preexisting fear memory at the initial phase of fear memory consolidation, the $\beta$ lac infusion immediately after training may not recover the anisomycininduced amnesia. Consistent with this idea, we found that the infusion of $\beta$ lac had no effect on the anisomycin-induced memory impairment during memory acquisition as well as the memory acquisition itself (Fig. 4B), even though the polyubiquitinated protein level increased after fear conditioning (fig. S5). Combined with the data in Fig. 3, these results support that the infusion of $\beta$ lac mainly suppresses the destabilizing process of the preexisting fear memory, and that protein synthesis in the hippocampus is required for the association of shock and context. The increase in polyubiquitination after conditioning may reflect the destabilization of preexisting synapses which were related to the context or shock but did not encode the contextual fear memory.

If the retrieval induces the destabilization of the preexisting memory, it would also occur in the extinction which is produced by repetitive retrievals in the absence of unconditioned stimuli. To test this idea, we performed the extinction training for two days with two spaced retrievals per day (24). Either vehicle or $\beta$ lac was infused immediately after the retrievals, and the freezing level was tested on the third day (Fig. 4C). Interestingly, ßlac infusions into area CA1 suppressed the extinction of contextual fear memory (Fig. 4D and fig. S4B). The freezing level was significantly reduced by the extinction in the animals re-exposed to the context with vehicle infusions whereas control animals without context reexposure showed no extinction (Fig. 4D and fig. S4B). Thus, 
our data suggest that ubiquitin/proteasome-dependent protein degradation is required for the memory extinction. This supports the idea that extinction is not only "inhibitory new learning” of a context-no shock association, but also involves at least some "unlearning" (or forgetting) of the preexisting context-shock association $(25,26)$. Furthermore, extinction has been suggested to involve memory updating process (26). Combined, our results support the idea that memory retrieval makes preexisting memory labile via ubiquitin/proteasomedependent protein degradation in order to update or reorganize the memory with new information.

Our data also showed that infusion of $\beta$ lac alone immediately after conditioning did not impair the acquisition of fear memory. This result disagrees with previous studies in some aspects $(14,27)$. In these studies, the consolidation of inhibitory avoidance or contextual fear memory is impaired by disturbance of ubiquitin/proteasome pathway. These discrepancies may reflect the differences in the experimental system, animal species or brain regions involved (28). It is known that the circuits involved in the processing of inhibitory avoidance task are somewhat different from the circuits of classical fear conditioning (29). Moreover, in contextual fear conditioning, three variables -re-exposure duration, the age of the memory and the strength of the memory- influence the memory processes activated during retrieval (2).

NMDA receptor activation triggers the destabilization of the consolidated fear memory (8). In cultured neurons, glutamatergic transmission activates ubiquitin/proteasome system $(30,31)$. It would be interesting to speculate that ubiquitin/proteasome-dependent protein degradation is increased by downstream signaling of NMDA receptor activation and destabilizes the retrieved fear memory.

We have shown that ubiquitin/proteasome-dependent degradation of preexisting postsynaptic proteins is involved in memory reorganization after retrieval. Our results support the idea that memory reorganization occurs via both degradation of preexisting synapses and synthesis of updated synapses. Preexisting memory may be rebuilt in conjunction with new information via the protein degradation and concurrent synthesis especially in the synaptic region.

\section{References and Notes}

1. K. Nader, G. E. Schafe, J. E. Le Doux, Nature 406, 722 (2000).

2. A. Suzuki et al., J. Neurosci. 24, 4787 (2004).

3. M. H. Milekic, C. M. Alberini, Neuron 36, 521 (2002).

4. P. W. Frankland et al., Learn. Mem. 13, 451 (2006).

5. C. M. Alberini, Trends Neurosci. 28, 51 (2005).

6. R. G. Morris et al., Neuron 50, 479 (2006).

7. Y. Dudai, Curr. Opin. Neurobiol. 16, 174 (2006).

8. C. Ben Mamou, K. Gamache, K. Nader, Nat. Neurosci. 9, 1237 (2006).
9. M. Eisenberg, T. Kobilo, D. E. Berman, Y. Dudai, Science 301, 1102 (2003).

10. O. Steward, E. M. Schuman, Annu. Rev. Neurosci. 24, 299 (2001).

11. M. D. Ehlers, Nat. Neurosci. 6, 231 (2003).

12. A. N. Hegde et al., Cell 89, 115 (1997).

13. A. N. Hegde, A. L. Goldberg, J. H. Schwartz, Proc. Natl. Acad. Sci. U.S.A. 90, 7436 (1993).

14. M. Lopez-Salon et al., Eur. J. Neurosci. 14, 1820 (2001).

15. R. Fonseca, R. M. Vabulas, F. U. Hartl, T. Bonhoeffer, U. V. Nagerl, Neuron 52, 239 (2006).

16. E. R. Kandel, Science 294, 1030 (2001).

17. A. Ciechanover, Nat. Rev. Mol. Cell. Biol. 6, 79 (2005).

18. Q. Deveraux, V. Ustrell, C. Pickart, M. Rechsteiner, J. Biol. Chem. 269, 7059 (1994).

19. K. Ferrell, Q. Deveraux, S. van Nocker, M. Rechsteiner, FEBS Lett. 381, 143 (1996).

20. C. Luscher, R. A. Nicoll, R. C. Malenka, D. Muller, Nat. Neurosci. 3, 545 (2000).

21. S. Lim et al., J. Biol. Chem. 274, 29510 (1999).

22. J. A. Lee et al., J. Cell Biol. 174, 827 (2006).

23. T. Abel, K. C. Martin, D. Bartsch, E. R. Kandel, Science 279, 338 (1998).

24. K. M. Lattal, T. Abel, J. Neurosci. 21, 5773 (2001).

25. K. M. Myers, M. Davis, Neuron 36, 567 (2002).

26. J. Ji, S. Maren, Hippocampus 17, 749 (2007).

27. Y. H. Jiang et al., Neuron 21, 799 (1998).

28. S. J. Martin, P. D. Grimwood, R. G. Morris, Annu. Rev. Neurosci. 23, 649 (2000).

29. A. E. Wilensky, G. E. Schafe, J. E. LeDoux, J. Neurosci. 20, 7059 (2000).

30. B. Bingol, E. M. Schuman, Nature 441, 1144 (2006).

31. L. Guo, Y. Wang, Neuroscience 145, 100 (2007).

32. We thank Eric Kandel, Alcino Silva, Paul Frankland, Sheena Josselyn and Yong-Seok Lee for reading the manuscript and critical discussion. We are grateful to lab members for their technical help. We also thank Eunjoon Kim for providing Shank antibody. This work was supported by the Creative Research Initiative Program of the Korean Ministry of Science and Technology (to B.K.K.).

\section{Supporting Online Material}

www.sciencemag.org/cgi/content/full/1150541/DC1

Materials and Methods

Figs. S1 to S5

References

14 September 2007; accepted 11 January 2008

Published online 7 February 2008;10.1126/science.1150541

Include this information when citing this paper. 
Fig. 1. Fear memory retrieval increases poly-ubiquitination in the hippocampal synaptosomal membrane fraction. (A) The schematic experimental procedure used for examining the level of poly-ubiquitination after retrieval. One day after contextual fear conditioning, mice were re-exposed to the context to retrieve the fear memory. $15 \mathrm{~min}$ or $60 \mathrm{~min}$ after retrieval, hippocampi were isolated and poly-ubiquitination assay was performed. (B) Representative data from polyubiquitination assay show the total poly-ubiquitinated proteins which were pulled down by GST-S5a and detected by anti-ubiquitin antibody ( $\alpha$-Ub). The poly-ubiquitination level is increased $15 \mathrm{~min}$ and $60 \mathrm{~min}$ after retrieval when it is compared with the level in Nv (naïve: the mice group which received only handling) or No $\mathrm{R}$ (no retrieval: the mice group of which hippocampi were isolated without retrieval). Polyubiquitinated proteins were not bound to GST alone. Input indicates an aliquot of total ubiquitinated proteins in each extract.

Fig. 2. Retrieval stimuli regulate the degradation of specific postsynaptic proteins. (A and B) Poly-ubiquitinated Shank and GKAP, but not PSD-95, are significantly increased 1h after retrieval. (A) Representative data show the level of Shank (upper panel), GKAP (middle panel), and PSD-95 (lower panel) in the isolated poly-ubiquitinated proteins from the hippocampal synaptosomal fraction. Poly-ubiquitinated proteins were isolated by the same experimental protocol in Fig. 1. (B) Bars represent the means \pm SEM of the percent change of poly-ubiquitinated Shank and GKAP proteins ( ${ }^{*} P$ $<0.05$; unpaired t-test; $n=3$ for each group). (C to $\mathbf{E}$ ) Dynamics of endogenous Shank protein level following retrieval. (C) The protein expression level of Shank isoforms (160 kDa: upper band, $130 \mathrm{kDa}$ : lower band) in the hippocampal synaptosomal membrane fraction was decreased up to $2 \mathrm{~h}$ after retrieval, but it was restored to the basal level at $6 \mathrm{~h}$. (D and E) Bars represent the means \pm SEM of the quantified level of Shank isoforms. Both Shank isoforms were significantly reduced at $2 \mathrm{~h}$ after retrieval $(* P<0.05$, ${ }^{* *} P<0.01$; unpaired t-test; $n=4$ for each group).

Fig. 3. Proteasome inhibitor blocks the anisomycin-induced memory impairment after retrieval. (A) Schematic illustration showing the cannula locations in the hippocampus at three different rostral-caudal planes. Numbers indicate the posterior direction from the bregma. Yellow circles, green squares, blue squares, and red circles indicate the infusion areas of vehicle, anisomycin, $\beta$ lac, and anisomycin with $\beta$ lac, respectively. (B) Schematic view of the experimental procedure used for evaluating the effect of $\beta$ lac on the retrieval. (C) Bars represent the means \pm SEM of the freezing level at retrieval 2. Compared with the vehicle-infused control group (veh, $n=11$ ), $\beta$ lac infusion ( $\beta$ lac, $n=10$ ) alone had no effect on the fear memory retrieval while anisomycin- infused group (ani, $n=14$ ) showed impaired fear memory retrieval. However, concurrent infusion of $\beta$ lac with anisomycin (ani+ $\beta$ lac, $n=10$ ) prevented the impairment of fear memory retrieval induced by anisomycin $(* P<0.05$; one-way ANOVA and Newman-Keuls multiple comparison test). (D) Bars represent the means \pm SEM of the relative freezing level of retrieval 2 ((R2 / R1) x 100 (\%)). Dotted line indicates the freezing level of retrieval 1 . Significant reduction in the freezing level at the anisomycin-infused group was reversed by the co-infusion of $\beta$ lac $(* * * P<0.001$; one-way ANOVA and Newman-Keuls multiple comparison test).

Fig. 4. Protein degradation is not required for the memory consolidation whereas it is critical for the memory extinction. (A) Schematic view showing the protocol of behavioral experiments to examine the effect of $\beta$ lac on the fear memory acquisition. (B) Bar graph represents the mean \pm SEM of the freezing level at the retrieval day. Single infusion of $\beta$ lac $(n=$ 12 ) had no effect on the fear memory formation compared with vehicle-infused control group $(n=11)$ whereas anisomycin-infused group $(n=14)$ showed significant impairment of the fear memory. Co-infusion of $\beta$ lac with anisomycin $(n=12)$ also had no effect on the anisomycininduced amnesia during memory acquisition ( ${ }^{*} P<0.05$; oneway ANOVA and Newman-Keuls multiple comparison test).

(C) Schematic view of the behavioral experiments performed to examine the effect of $\beta$ lac on the fear memory extinction.

(D) Fear memory extinction is impaired in the $\beta$ lac-infused group ( $n=7$ ) compared to the vehicle-infused group (veh, $n=$ 7). Control group $(n=8)$ indicates the vehicle-infused animals without extinction training ( ${ }^{*} P<0.05$; one-way ANOVA and Newman-Keuls multiple comparison test). 


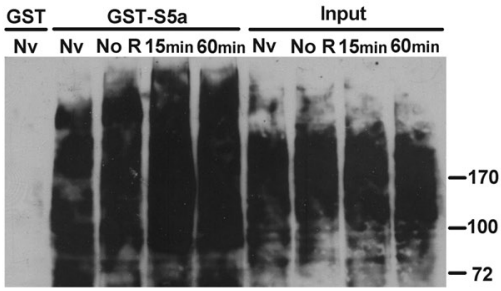

IB: $\alpha-U b$ 
B
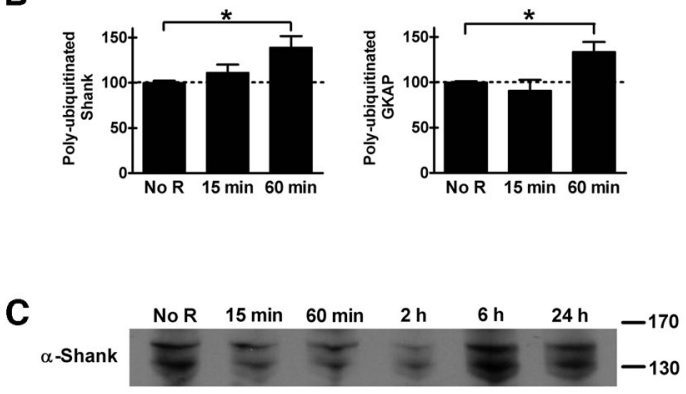

D

$160 \mathrm{kDa}$

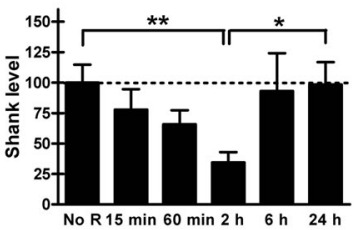

E

$130 \mathrm{kDa}$

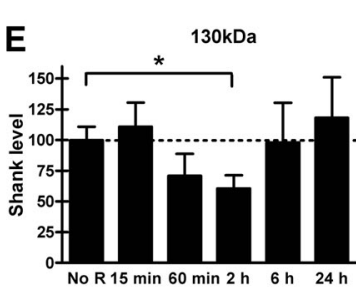

IB: $\alpha-P S D-95$

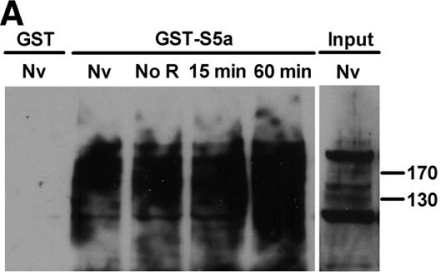

IB: $\alpha$-Shank

IB: $\alpha$-GKAP $-130$

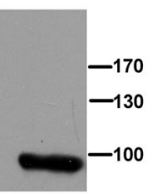


Bregma

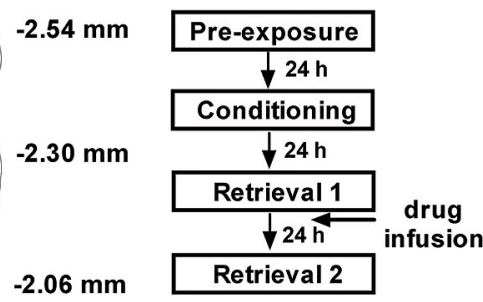

C

D

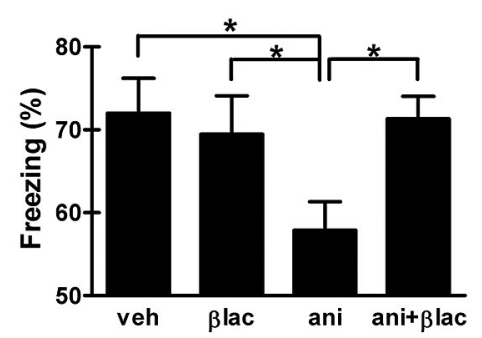

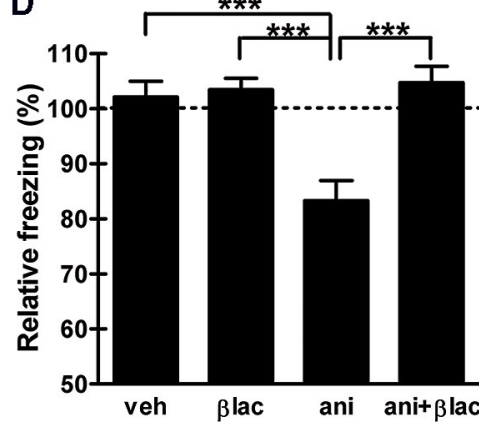


